\title{
NUEVAS APORTACIONES AL ESTUDIO POLÍNICO DE SEDIMENTOS DEL PARQUE NATURAL DE LOS ALCORNOCALES (CÁDIZ, SUR DE ESPAÑA)
}

\author{
Ángeles GUTIÉRREZ, M. Josefa DÍEZ, Marta NEBOT y Manuel CELIS.
}

\begin{abstract}
RESUMEN. Nuevas apotaciones al estudio polínico de sedimentos del Parque Natural de los Alcornocales (Cádiz, Sur de España). En este trabajo se completa el contenido polínico de una columna de sedimento de $300 \mathrm{~cm}$, extraida de una zona semiturbosa de la Sierra del Aljibe en el Parque Natural de los Alcornocales (Cádiz). Se discuten las variaciones polínicas porcentuales de los taxones identificados, consideradas de nuevo aquí a la luz de los nuevos datos, fundamentalmente la datación con radiocarbono, y se interpretan los diagramas polínicos resultantes en términos paleoecológicos. Por otro lado, y en base a estudios previos en el norte de Marruecos, zona de características ecológicas e históricas similares a la zona estudiada, se concluye el uso diferente del territorio a ambos lados del estrecho.
\end{abstract}

Palabras clave. Palinología, Paleoecología, Holoceno, Subatlántico, Sur de España.

SUMMARY. New contributions to pollen analysis from the Parque Natural de los Alcornocales (Cádiz, south Spain). This paper completes the pollen analysis of a $300 \mathrm{~cm}$-deep peaty deposit in Sierra del Aljibe. Alcornocales Natural Park. The pollen percentage diagram is discussed again in terms of palaeoecology, to wich radiocarbon dating contributes especially. From the results, together with those from a review of literature of studies of northern Morocco (with similar ecological and historical feature to the area studied), it is concluded that there is a different territorial custom on both side of the strait.

Key words. Palynology, Palaeoecology, Holocene, Sub-Atlantic, South Spain.

\section{INTRODUCCIÓN}

Los resultados paleopalinológicos desempeñan un papel fundamental en la interpretación de la historia de la vegetación. No obstante, los espectros polínicos pueden verse afectados por diversos factores, como la preservación diferencial de los palinomorfos implicados (Havinga, 1964), las diferencias específicas en las capacidades de producción y dispersión polínicas (Faegri e Iversen, 1975), etc. Es, por tanto, recomendable realizar estudios de lluvia polínica actual a fin de poner de manifiesto la intervención eventual de dichos 
factores y sus condicionantes (Horowitz, 1992). A la vez se pueden realizar estudios tafonómicos donde se analice la incorporación, distribución, alteración y desplazamiento postdeposicionales (Moore et al., 1991). Estos estudios complementarios son necesarios, pues un espectro polínico aislado no traduce necesariamente la imagen de un tipo de vegetación existente en el pasado. Finalmente, el cálculo de la concentración polínica puede ser un buen indicador de las variaciones en la cobertura vegetal siempre que no haya grandes variaciones en la velocidad de formación del depósito (Moore et al., 1991).

Cuando sea posible, los resultados polínicos deben apoyarse además en los de otras disciplinas que aporten datos complementarios y/o abran nuevas perspectivas de discusión. Las más utilizadas son la arqueología, la paleobotánica, la sedimentología, la paleontología y la geocronología. Esta última con especial relevancia, pués pretende establecer la edad absoluta del proceso tafonómico, fundamentalmente con el empleo de isótopos de carbono, oxígeno, torio-uranio, potasioargón u otros.

Este estudio se ha realizado en el Parque Natural de los Alcornocales (fig. 1), situado en la zona norte del Estrecho de Gibraltar, dentro de la provincia de Cádiz, incluido en el Subsector biogeográfico español Aljíbico (Rivas-Martínez, 1988), y cuyas características generales fueron descritas en un trabajo previo (Gutiérrez et al., 1996).

Este trabajo, junto al mencionado anteriormente (Gutiérrez et al., l.c.), son los únicos conocidos referentes al Cuaternario en el área de estudio, quizá debido a la escasez de depósitos organogénicos adecuados como turbas, lagunas, etc. Además de los existentes en el norte de Marruecos (Reille, 1977) se tienen datos del suroeste peninsular (Stevenson, 1984; 1985a; 1985b; Stevenson y Moore, 1985, 1988; Stevenson y Harrison, 1992), pero en un

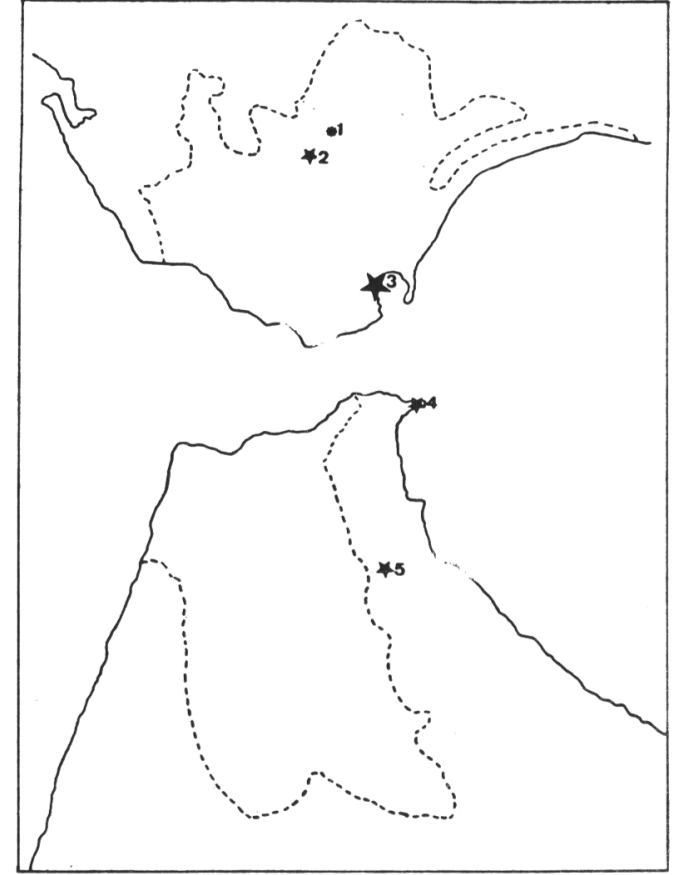

Figura 1. Localización del punto de muestreo (1) y su posición con respecto al norte de África. La línea discontínua representa el Subsector biogeográfico español Aljíbico (Rivas-Martínez, 1988). (2) Acalá de los Gazules; (3) Algeciras; (4) Ceuta; (5) Chefchaouen. Location of sampling point (1) and its position with respect to northern Africa. The broken line shows the Aljibico Spanish biogeographic subsector (Rivas Martinez, 1988). (2) Alcalá de los Gazules; (3) Algeciras; (4) Ceuta; (5) Chefchaouen.

marco diacrónico diferente.

Las investigaciones en curso, que incluyen una secuenciación radiocarbónica detallada y un mayor número de análisis polínicos, determinarán el control bioestratigráfico y cronológico del registro.

\section{MATERIAL Y MÉTODOS}

La localización del lugar de muestreo se refleja en la figura 1. Las «lagunas» de la Sierra del Aljibe se localizan en la raiz del 
Estudio polínico P.N. Alcornocales (Cádiz)

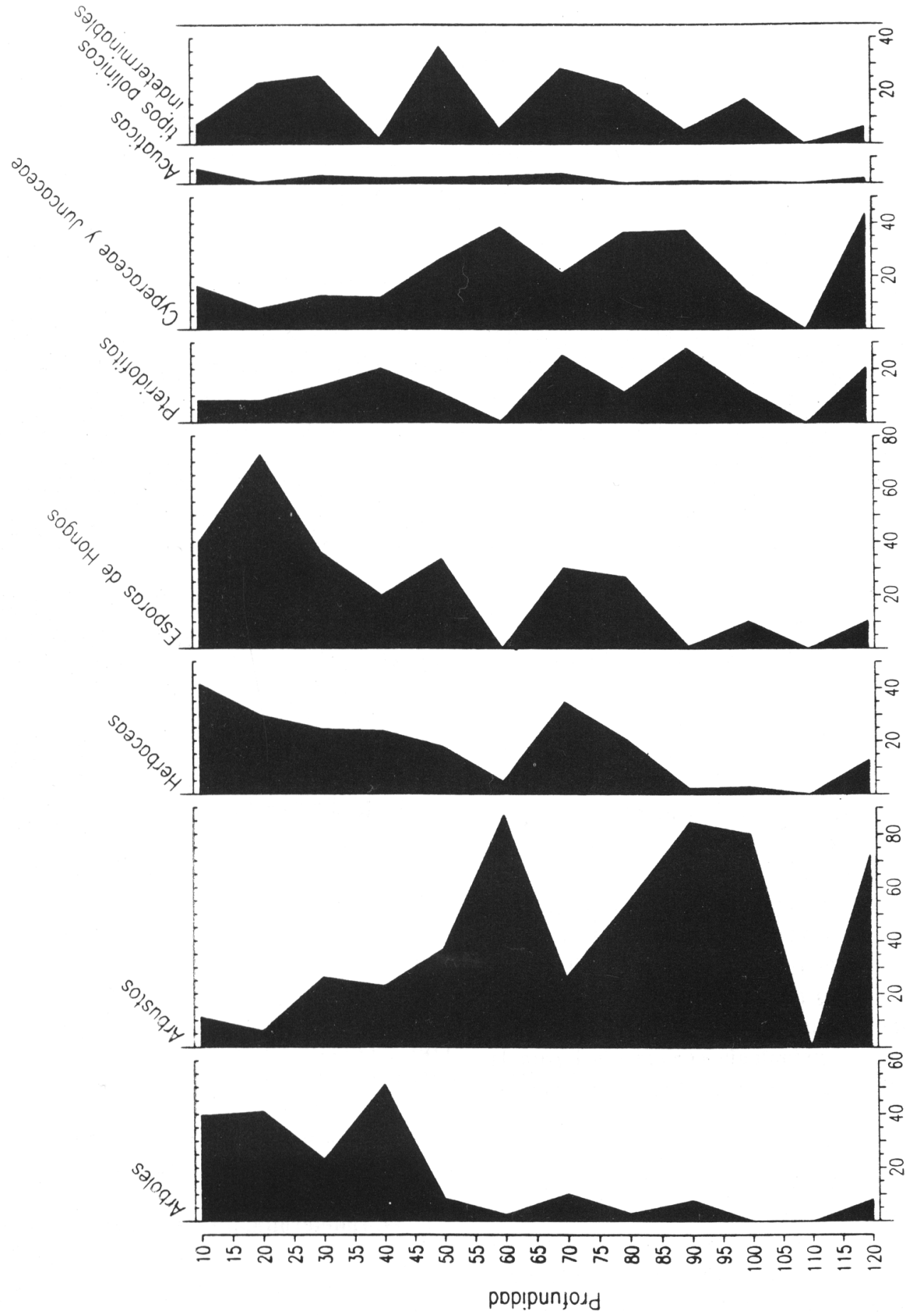

Figura 2. Diagrama polínico sintético. Synthetic pollen diagram. 
piedemonte occidental del Picacho. Su génesis está relacionada con dos factores: la existencia de una cobertura detrítica de arenas móviles y finas sobre sustratos arcillosos rojizos del Mioceno de carácter impermeable, y la propia planitud de la superficie del piedemonte.

La descripción de la recogida de muestras, así como el tratamiento del sedimento fue descrito con precisión en un trabajo anterior (Gutiérrez et al., 1996).

La edad absoluta más antigua del sedimento sólo se ha podido obtener a los 60 $\mathrm{cm}$ de profundidad, ya que por la naturaleza del mismo (areniscas), y el material disponible (sonda de media caña), fue necesario abrir una cata para obtener la cantidad suficiente de sedimento para su datación con radiocarbono, encontrándose la capa freática a $\operatorname{los} 70 \mathrm{~cm}$ de profundidad.

\section{RESULTADOS Y DISCUSIÓN}

Como ya se mencionó en el trabajo anterior (Gutiérrez et al., 1996) las muestras analizadas por debajo de los $120 \mathrm{~cm}$ de profundidad resultaron estériles polínicamente.

Los resultados de la datación absoluta con $\mathrm{C}^{1+}$ mostraron una antiguedad de $1.200 \pm$ $70 \mathrm{BP}$ y $\mathrm{C}^{13} / \mathrm{C}^{12}=-25 \%$ a $\operatorname{los} 60 \mathrm{~cm} \mathrm{de}$ profundidad, por lo que podría estimarse que la antigüedad de la columna $(120 \mathrm{~cm}$ de profundidad) no supera los 2000-2500 años; estaríamos por tanto dentro del Subatlántico según la terminología convencional para la estratigrafía holocénica (Reille y Andrieu, 1991).

En la figura 2 se representan los datos obtenidos referentes a polen arbóreo, arbustivo y herbáceo, esporas de hongos y pteridofitas y polen de Cyperaceae-Juncaceae-acuáticas. El polen de Cyperaceae-Juncaceae-acuáticas, así como las esporas de hongos y pteridofitas han sido excluidos del diagrama general de tipos polínicos (fig. 3) por ser estas especies actualmente abundantes en el lugar de muestreo, pudiendo por ello aparecer sobrerrepresentadas.

La figura 3 refleja los distintos tipos polínicos arbóreos, arbustivos y herbáceos, así como sus porcentajes.

Los tipos polínicos identificados pueden organizarse en seis grupos:

I. Asociados a zonas húmedas y/o acuáticas y esporas de hongos: esporas de Basidiomicetos fundamentalmente y pteridofitas (Isoetes histrix, I. velata y Asplenium), así como polen de CyperaceaeJuncaceae-acuáticas, mayoritariamente Luzula, pero también Cyperus longus, Juncus, Ruppia y Potamogeton pectinatus (fig. 4).

II. Arbóreos: Tipo Quercus suber, Tipo Pinus pinea, Corylus avellana y Celtis australis.

III. Brezos: E. arborea, E. scoparia, E. erigena y E. australis.

IV.Ruderales (Reille, 1977; Behre, 1981; y Dimbleby, 1985): Tipo Fumaria officinalis, Tipo Papaver rhoeas, Tipo Silene latifolia, Tipo Sinapis arvensis, Tipo Conium maculatum, Umbelliferae (Bupleurum o Conium), Tipo Echium plantagineum, Tipo Anthemis arvensis, Tipo Centaurea calcitrapa, Tipo Crupina vulgaris, Tipo Lactuca serriola, Tipo Senecio vulgaris, Tipo Leucojum autumnale, Tipo Narcissus papyraceus.

V. Casos especiales: Annonaceae, Dicotiledónea primitiva y Ephedra fragilis.

VI. Indeterminables: en este apartado se incluyen granos de polen que por su estado de deterioro no se han podido incluir en ningún grupo concreto con suficiente garantía. (Birks y Birks, 1980, sec. Martín Consuegra, 1993). 


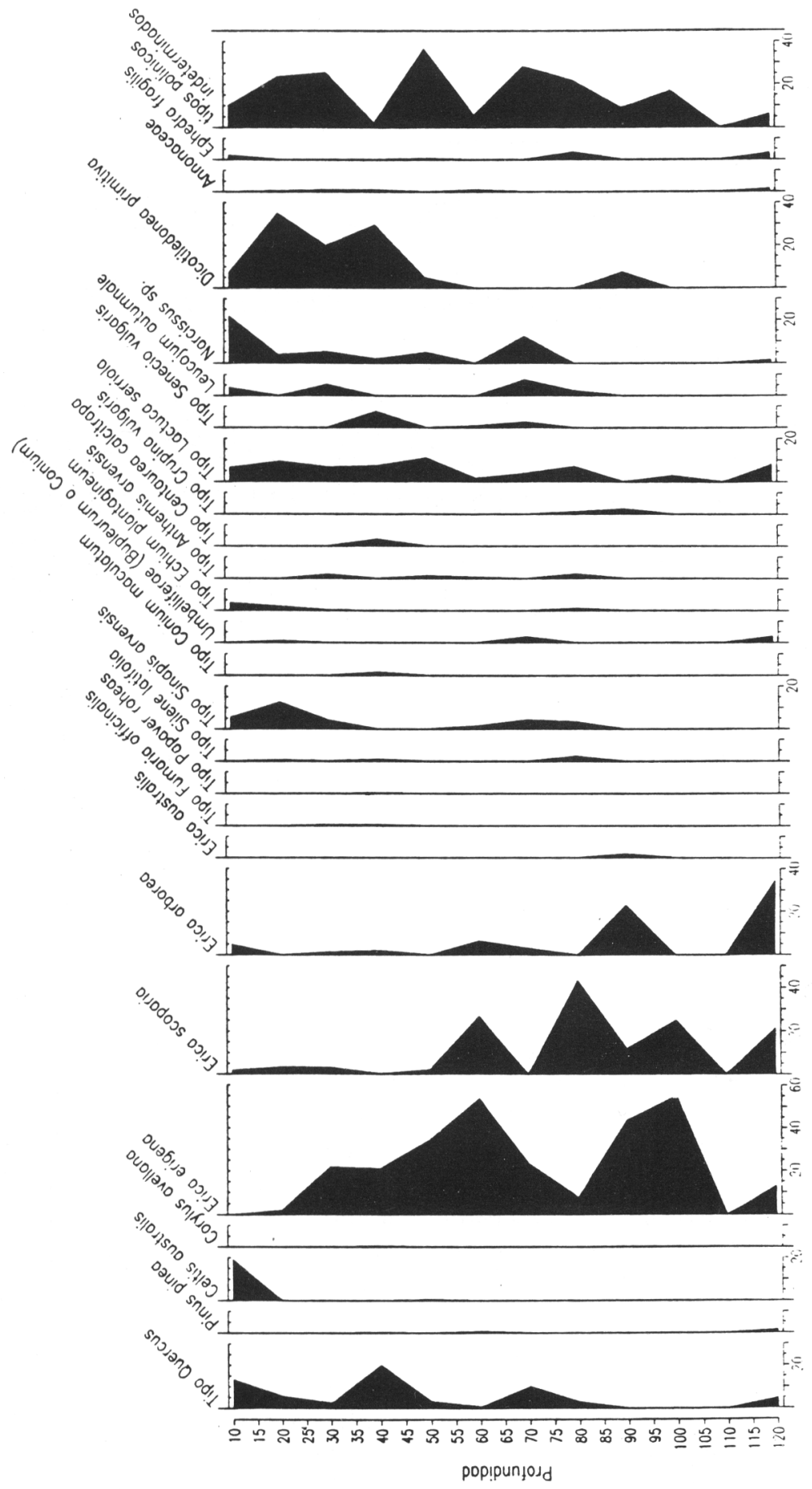

Figura 3. Diagrama polínico de los tipos polínicos encontrados a lo largo de los $120 \mathrm{~cm}$ de profundidad de la columna analizada. Pollen diagram of pollen types found throughout the $120 \mathrm{~cm}$ of the studied column. 


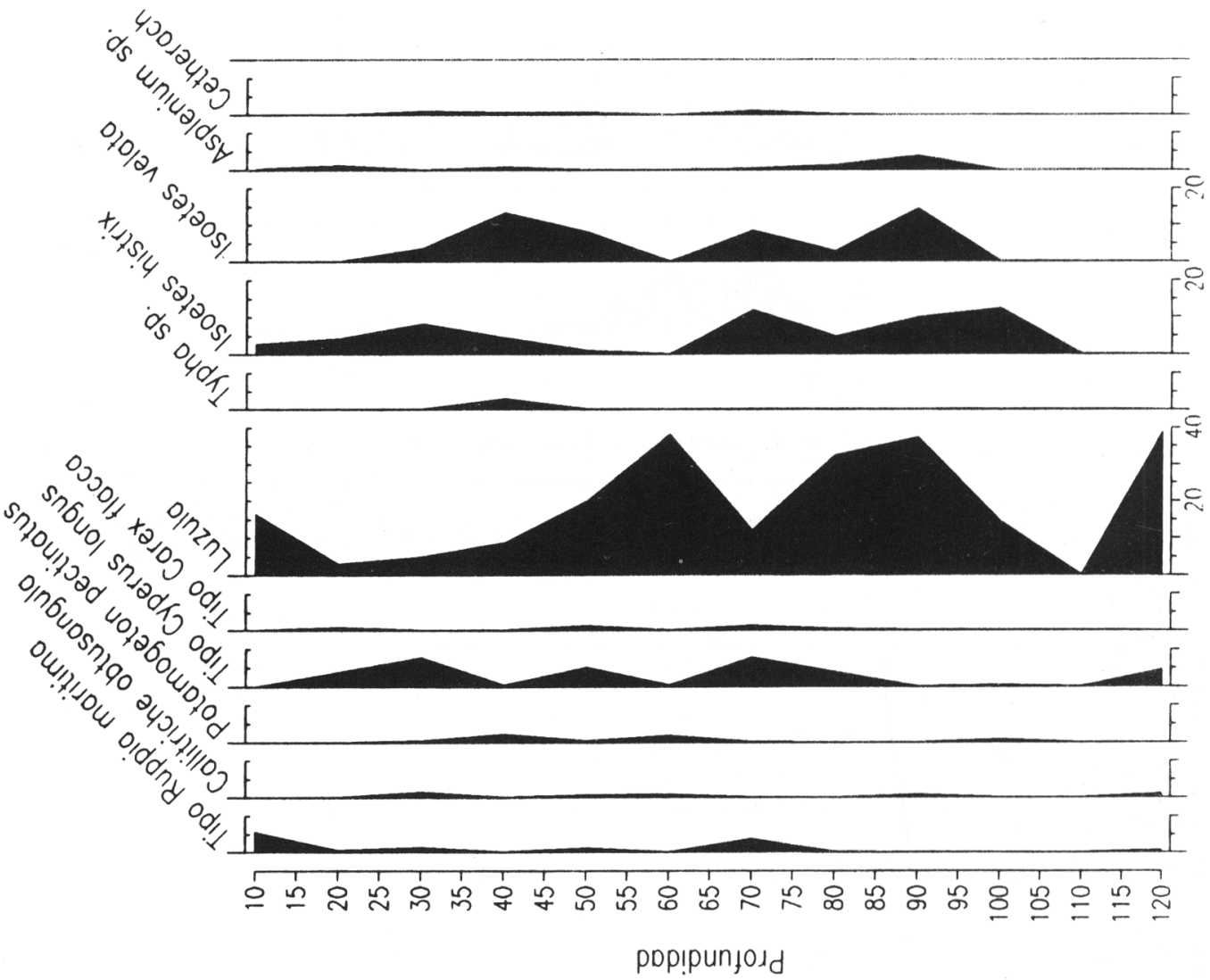

Figura 4. Diagrama polínico de las especies acuáticas y asociadas a ambientes húmedos. Pollen diagram of aquatic species and those associated to wet environments.

Como se puede observar en la figura 2, las esporas de hongos, pteridofitas y en general especies asociadas a ambientes húmedos (Grupo I), están sobrerrepresentadas. Su variación comparada sigue un cierto paralelismo en el diagrama, en el que a partir de los $50 \mathrm{~cm}$ se observa una disminución del polen en general y de las especies acuáticas, así como un aumento de las esporas de hongos (fig. 2). Estos grupos no tienen más que un significado puramente edáfico, por lo que ésto podría interpretarse como una desecación de la laguna, conservándose un alto grado de humedad en el terreno, tal como se observa en la actualidad.

La valoración que nos merecía la baja representación del polen arbóreo (PA) (Grupo II, figs. 2 y 3 ) se realizó en el trabajo anterior (Gutiérrez et al., l.c.), en parte por lo llamativo de su porcentaje. No obstante, aquí se refleja con mayor claridad, al compararlo con el total de grupos polínicos encontrados en la columna, mediante una visión de conjunto más precisa ofrecida en la figura 2. Además de la sabida infrarrepresentación, y a la vista de la figura 3, llama especial atención la escasa proporción de especies autóctonas (fig. 3). No obstante, puede observarse cómo por encima de los 60 $\mathrm{cm}$, o lo que es lo mismo, a partir del siglo VIII (fig. 2) el porcentaje de polen arbóreo se incrementa. Generalmente se piensa que el aspecto de buena conservación de enclaves 
como la Sierra del Aljibe es más el resultado de un abandono posterior y relativamente reciente que de su carácter de zona inalterada (Parra, 1987).

La abundancia de polen de Brezos y sus correspondientes diagramas fueron comentados anteriormente por Gutiérrez et al. (1996). No obstante, a raiz de la datación con ${ }^{14} \mathrm{C}$ se concluye que la abundancia de Erica erigena por debajo de los $60 \mathrm{~cm}$, así como su paralelismo con el incremento de especies acuáticas, implica una mayor extensión de la laguna antes del siglo VIII.

El grupo mejor representado en cuanto a número de taxones es el de los ruderales (Grupo IV), aunque sus porcentajes en la mayoría de los casos son pequeños. No obstante, la ruderalización comienza a ser más evidente a partir de los $90 \mathrm{~cm}$ (fig. 3), donde empieza a apreciarse con más frecuencia. En cualquier caso la mayoría de taxones encontrados son de difícil interpretación paleoecológica (Tipo Senecio vulgaris, Tipo Anthemis arvensis, Tipo Crupina vulgaris, etc.).

Dentro del grupo $\mathrm{V}$ dos taxones se han identificado con baja resolución: Annonaceae y Dicotiledonea primitiva. El interés de estos taxones, y la información que podrían aportar, está en espera de una identificación más precisa. No obstante debido al interés de los mismos se han considerado en el diagrama y separados, junto con Ephedra, género comentado con anterioridad (Gutiérrez et al., l.c.).

Según los trabajos de Reille en el norte de Marruecos, y aparte de lo ya mencionado por Gutiérrez et al. (l.c.), otra diferencia fundamental que se pone de manifiesto entre ambas zonas es el uso diferente del territorio, ya que en el norte de Marruecos se observa un retroceso de los bosques de Quercus, que en general coincide con la introducción del cultivo del olivo, concretamente en el siglo X, coincidiendo con la invasión árabe (Reille, 1977), mientras que en la Sierra del Aljibe se incrementa en general el polen de Quercus y no se detecta la presencia del olivo.

\section{CONCLUSIONES}

La desecación de la laguna comenzó después del siglo VIII.

A partir del siglo VIII se incrementa el porcentaje de polen arbóreo, confirmándose la opinión generalizada de que el aspecto de buena conservación de enclaves como la Sierra del Aljibe es más el resultado de un abandono posterior y relativamente reciente que de su carácter de zona inalterada.

La evolución del polen de las especies de Erica junto con el de especies acuáticas indica una mayor extensión de la laguna en el siglo VIII.

La evolución del polen de Quercus y su comparación con datos del norte de Marruecos pone de manifiesto el uso diferente del territorio a ambos lados del estrecho.

AGRADECIMIENTOS. Los autores desean expresar su agradecimiento a los doctores Juán Arroyo y J. S. Carrión por sus comentarios y ayuda en la realización de este trabajo.

\section{BIBLIOGRAFÍA}

BEHRE, K.E. -1981- The interpretation of anthropogenic indicators in pollen diagrams. Pollen et Spores 23(2): 225-245.

DIMBLEBY, G.W. -1985- The palynology of archaeological sites. Academic Press Inc. London.

FAEGRI, K. \& J. IVERSEN -1975- Textbook of Pollen Analysis. Blackwell Scientific Publications Oxford.

GUTIÉRREZ, A., M. NEBOT y M. J. DÍEZ -1996Introducción al estudio polínico de sedimentos del Parque Natural de los Alcornocales. Almoraima 15: 87-90.

HAVINGA, A.J. -1994- Investigations into the differential corrosion susceptibility of pollen and spores. Pollen et Spores 6 : 621-635.

HOROWITZ, A. -1992- Palynology of arid lands. Elsevier, Amsterdam.

MARTÍN-CONSUEGRA, E. -1993-Palinología y 
Botánica histórica del complejo de Madinat alZahra. Tesis Doctoral. Departamento de Biología Vegetal y Ecología. Universidad de Córdoba.

MOORE, P.D., J.A. WEBB \& M.E. COLLLINSON 1991-. Pollen analysis. 2nd Edition. Blachwell Scientific Publications, Oxford.

PARRA, F. -1987- Enciclopedia de la Naturaleza de España. Vol. 10. Monte Mediterráneo. Ed. Debate. Barcelona. pp. 12.

REILLE, M. - 1977- Contribution pollenanálytique à l'histoire holocène de la végétation des montagnes du Rif (Maroc Septentrional). Recherches Françaises sur le Quaternaire. INQUA. Supplément au Bulletin de l'Association Française pourl'Étude du Quaternaire 50: 5376.

REILLE, M. \& V. ANDRIEU -1991-Données nouvelles sur l'histoire postglaciaire de la végétation de Pyrenées occidentales (Francia). Centre de Recherches Académie de Sciences, Paris, t. 312, Série II. pp: 97-103.

RIVAS-MARTÍNEZ, S. -1988- Bioclimatología, biogeografía y series de vegetación de Andalucía Occidental. Lagascalia 15 (extra): 91-120.

STEVENSON, A.C. -1984-Studies in the vegetational history of S.W. Spain. III Palynological investigation at El Asperillo, Huelva. Journal of Biogeography 11: 527-551.

STEVENSON, A.C. -1985 a- Studies in the vegetational history of S.W. Spain. II Palynological investigation at Laguna de las Madres, S.W. Spain. Journal of Biogeography 12: 293-314.

STEVENSON, A.C. -1985 b- Studies in the vegetational history of S.W. Spain. I. Modern pollen rain in the Doñana National Park, Huelva. Journal of Biogeography 12: 243-268.
STEVENSON, A.C. \& R.J. HARRISON -1992Ancient forest in Spain: a model for land-use and dry forest management in South-west Spain from $4000 \mathrm{BC}$ to $1900 \mathrm{AD}$. Proceedings of the Prehistoric Society 58: 227-247.

STEVENSON, A.C. \& MOORE, P.D. -1985- Surface pollen and short core studies in Mediterranean heatland in southern Spain. Ecología Mediterranea XI (I): 129-133.

STEVENSON, A.C. \& MOORE, P.D. -1988-Studies in the vegetational history of S.W. Spain. IV Palynological investigation of a valley mire at El Acebrón, Huelva. Journal of Biogeography 15: 339-361.

Aceptado para su publicación en Abril de 1997

Dirección de los autores. Departamento de Biología Vegetal y Ecología. Facultad de Biología. Apdo. 1095. 41080. Sevilla. 